\title{
Access and closure management of large bore femoral arterial access
}

\author{
Amir Kaki MD ${ }^{1}$ | Nimrod Blank MD ${ }^{1}$ (D) $\quad$ M. Chadi Alraies $M^{1}$ (D) | \\ Marvin Kajy MD ${ }^{1}$ | Cindy L. Grines MD $^{2}$ | Reema Hasan $M^{3}$ । \\ Wah Wah Htun MD ${ }^{4}$ | James Glazier MD ${ }^{1}$ | Tamam Mohamad MD ${ }^{1}$ | \\ Mahir Elder MD ${ }^{1}$ | Theodore Schreiber MD ${ }^{1}$
}

\author{
${ }^{1}$ Wayne State University, School of Medicine, \\ Detroit Medical Center, Detroit Heart \\ Hospital, Detroit, Michigan \\ 2 Zucker School of Medicine at Hofstra \\ Northwell Health, Northshore University \\ Hospital, Manhasset, New York \\ 3 University of Michigan, Ann Arbor, Michigan \\ ${ }^{4}$ Northwell Health, Lenox Hill Hospital, New \\ York, New York \\ Correspondence \\ M. Chadi Alraies, MD, Wayne State \\ University, School of Medicine, Detroit \\ Medical Center, Detroit Heart Hospital, 311 \\ Mack Ave, Detroit, MI 48201. \\ Email: alraies@hotmail.com
}

Femoral and radial artery access continue to be the standard of care for percutaneous coronary interventions. Cardiac catheterization has progressed to encompass a wide range of diagnostic and interventional procedures including coronary, peripheral, endovascular, and structural heart disease interventions. Despite advanced technology to make these procedures safe, bleeding, and vascular complications continue to be a substantial source of morbidity, especially in patients undergoing large-bore access procedures. New variations of percutaneous devices have reduced complications associated with these procedures. However, safe vascular access with effective hemostasis requires special techniques which have not been well described in the literature. Large-bore femoral artery access is feasible, safe, and associated with low complication rates when a protocol is implemented. Wayne State University, Detroit Medical Center Heart Hospital is a tertiary care, high-volume center for endovascular, structural heart and complex high risk indicated procedures with more 150 procedures involving mechanical circulatory support (MCS) devices per year. In this manuscript, we describe our approach to femoral artery large-bore sheath insertion and management. Our protocol includes proper identification of the puncture site, device selection, insertion, assessment of limb perfusion while on prolong MCS support, and hemostasis techniques after sheath removal.

\section{KEYWORDS}

complex coronary intervention, coronary artery disease, femoral closure devices, interventional devices, large femoral access, structural heart disease

\section{1 | INTRODUCTION}

Successful cardiac catheterization begins with safe vascular access and ends with effective hemostasis after equipment removal. Over the past years, cardiac catheterization has progressed to encompass a wide

Abbreviations: CHIP, complex high risk indicated patients; EVAR, endovascular aortic repair; MCS, mechanical circulatory support; TAVR, transcatheter aortic valve replacement. range of diagnostic and interventional procedures including coronary, peripheral, endovascular, and structural heart disease interventions. Vascular access has evolved from an approach requiring surgical cutdown in the 1940's with primitive catheters to current percutaneous techniques using advanced catheters, guides, wires, and closure devices that allow safe arteriotomy and effective closure. ${ }^{1-3}$

Despite multiple adjustments in devices and techniques, bleeding, and vascular complications continue to be a substantial source of 
morbidity especially in patients undergoing large-bore access procedures. ${ }^{4-6}$ Although femoral access is the most common approach in the United States, adoption of transradial access has been increasing. ${ }^{7-9}$

Over the years, elderly patients with multiple comorbidities are increasingly referred for high risk structural and endovascular interventions that often require mechanical circulatory support (MCS). These new and advanced technologies require large-bore arterial accesses. ${ }^{10,11}$

This article will address large-bore sheath insertion, maintenance, and removal. Techniques for the maintenance of lower limb perfusion during large-bore sheath access and the management of vascular complications will also be discussed in detail.

\subsection{Impact of peripheral vascular disease on vascular access}

The size of the iliac and common femoral arteries varies greatly, depending mainly on the patient body habitus and may be affected by the extent of atherosclerosis and tortuosity. ${ }^{12,13}$ Peripheral vascular disease (PVD) and coronary artery disease (CAD) have similar risk factors, and thus, it is common to encounter the challenge of treating structural or complex high-risk indicated patients (CHIP) with concomitant significant PVD. ${ }^{14,15}$ Small caliber common femoral and iliac arteries (less than $5.5 \mathrm{~mm}$ ) with significant PVD increase the risk for vascular complications. The risk increases even more when larger bore sheaths are used. ${ }^{6,16-18}$ In some patients, femoral vascular access is prohibitive or carries extreme risk due to small caliber, complete occlusion, or severe calcification. This is often discovered at the time of procedure as patient presenting with acute coronary syndrome or cardiogenic shock that requires immediate attention and access for mechanical support. In these cases, alternative arterial access sites such as axillary artery, carotid artery, subclavian artery, transcaval, and direct transapical approaches. ${ }^{19-24}$

\section{2 | Mechanical circulatory support (MCS) devices}

Temporary percutaneous MCS devices are indicated for patients presenting with cardiogenic shock and those who are undergoing highrisk coronary interventions. Based on the amount of hemodynamic support required, operators have different percutaneous MCS devices which provide adequate circulatory support during high risk procedures. Venoarterial extracorporeal membrane oxygenation (VAECMO) and TandemHeart (centrifugal-flow pump, TandemLife, Pittsburgh, PA) are available in the United States. Both devices require two large-bore cannulas, one inserted into the vein (and into the left atrium via transseptal puncture for TandemHeart) and another into the artery. ECMO and TandemHeart devices require an arterial cannula ranging between 15-20 Fr. MCS that requires arterial access only include PulseCath and Impella. PulseCath uses an intra-aortic balloon pump console and requires a $19 \mathrm{Fr}$ sheath. This device provides approximately $2 \mathrm{~L} / \mathrm{min}$ flow. The Impella 2.5 and CP devices (Abiomed Inc., Danvers, MA) are FDA-approved for up to 6 days for cardiogenic shock and up to $6 \mathrm{~h}$ for high risk coronary interventions. The Impella
2.5 and CP provide direct cardiac unloading and antegrade flow of up to 2.5 and $4.0 \mathrm{~L} / \mathrm{min}$, respectively. These two devices require a single arterial access of $13 \mathrm{Fr}$ and $14 \mathrm{Fr}$, respectively.

\section{3 | Access site selection}

For advanced interventional procedures, multiple access sites are often needed. In these scenarios, a combination of transradial and femoral approach is utilized. Whereby the transradial approach is used to perform coronary interventions and the femoral artery is used to deploy a temporary percutaneous MCS device or perform structural interventions. In patients who are scheduled for such advanced procedures, the anatomy of at least one of the ilio-femoral arteries is known from previous procedures or previous CT studies or iliofemoral arteries. This allows the operator to plan the optimal arteriotomy site prior to the procedure by selecting access site and proper sheath size. An ultrasound-guided arterial puncture technique is recommended to ensure good visualization of the arterial puncture site. This may be achieved with fluoroscopy or ultrasound. The fluoroscopy-guided technique is the most frequently used. This technique initially calls for palpation of anatomic landmarks and pulse. Then, a radiopaque object is placed at the body surface and used as a reference to identify the location of the femoral head landmarks. ${ }^{11,25,26}$ Ultrasound-guided access can be used to improve arterial puncture safely by appropriately targeting the puncture at the level of the common femoral artery (CFA) and thereby avoiding puncture of the profunda, the superficial femoral arteries and disruption of a heavily calcified area. Seto et $\mathrm{al}^{27}$ showed that routine real-time ultrasound guidance improved CFA cannulation only in patients with high CFA bifurcations but reduced the number of attempts, time to access, risk of venipunctures, and vascular complications in femoral arterial access. Hind et $\mathrm{al}^{28}$ suggested that ultrasound guidance access results in more precise CFA anterior wall puncture and may result in fewer post-therapy complications when compared to fluoroscopy.

After the first arterial access is established, performing angiography of the iliac bifurcation with right and/or left iliac arteries to define the access site and vessels anatomy is recommended (Figure 1). This approach is also used to define the anatomy of the contralateral vessel using $10 \mathrm{~mL}$ of contrast media injected from contralateral side using 5 or 6-French IMA catheter and eventually the artery away from calcification and in the middle third of the femoral head. It is important to study the vessel size, degree of vessel tortuosity as well as calcification. Favorable features include iliac arteries diameter of $5.5 \mathrm{~mm}$ or more with minimal tortuosity and/or atherosclerosis. Alternatively, an iliac diameter of less than $5 \mathrm{~mm}$ with high tortuosity and significant atherosclerosis may preclude from using the vessel for large-bore ( $>10$-French) sheath access. Although rarely used at our institution, intravascular ultrasound (IVUS) may provide excellent assessment of the vessel anatomy including a detailed view of a diseased artery, including vessel size, plaque volume, calcification, and tortuosity and has an added value of reducing the need for contrast.

Planning access for TAVR requires knowledge of the luminal size as well as the degree of vessel calcification and tortuosity. Ramlawi et $\mathrm{al}^{29}$ 


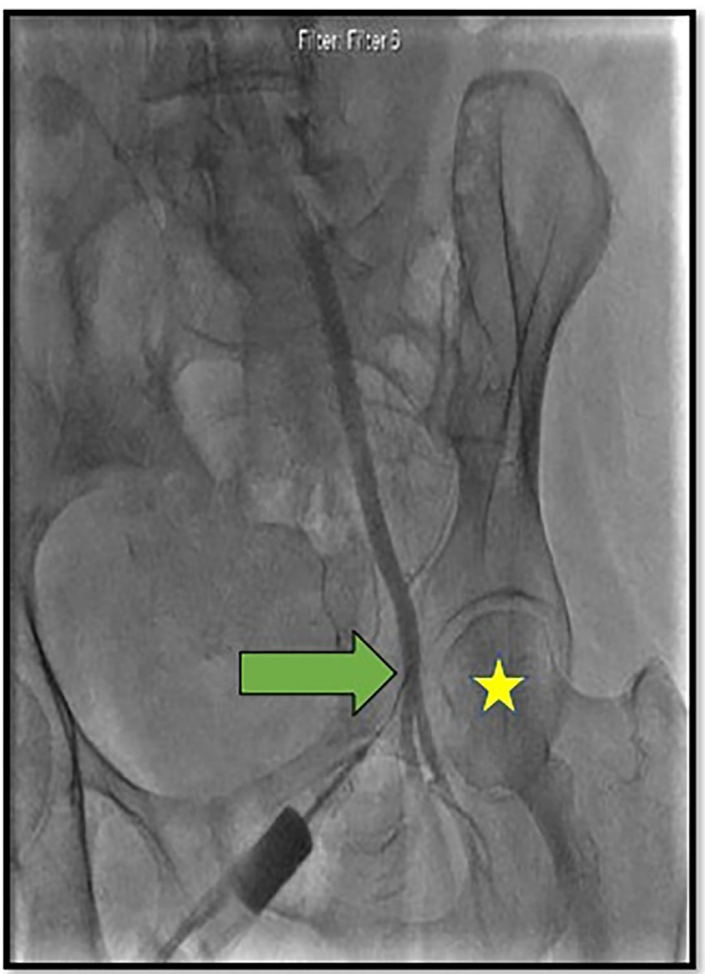

FIGURE 14 Fr left common femoral arterial access using micropuncture (green arrow). Notice the access point relation with the femoral head (asterisk). Left iliac-femoral angiography with no signs of atherosclerosis or stenosis

consider a high-quality thin-slice CT scan with contrast that extends from the femoral artery to the subclavian artery the cornerstone of evaluation. Also, CT angiography of the iliofemoral vasculature enables evaluation of vessel tortuosity, calcifications, and vessel diameters and may thereby inform decisions on the most suitable interventional approach.

\subsection{Femoral artery large size sheath insertion techniques}

Using ultrasound or direct fluoroscopy technique, a micropuncture needle is used to access the CFA. 6-French sheath then advanced over the micropuncture wire. Through the 6-French sheath, a stiff 0.035 wire (Supra Core or Lunderquist) wire is advanced and two Perclose ProGlide suture-mediated closure systems are deployed at $10^{\prime}$ and $2^{\prime}$ o'clock position. This facilitates future successful closure of the largebore puncture site. ${ }^{30,31}$ Subsequently, a sequential upsizing of the arterial puncture is performed with a series of dilators over a stiff 0.035 wire, prior to placement of the large sheath.

\section{5 | Maintaining lower extremity perfusion}

After the large-bore sheath is positioned and secured, it is crucial to assess vessel patency and assure preserved distal perfusion into the extremity (Figure 2). An aorto-iliac angiogram is performed by contrast injection through the large-bore sheath's side arm or by using selective

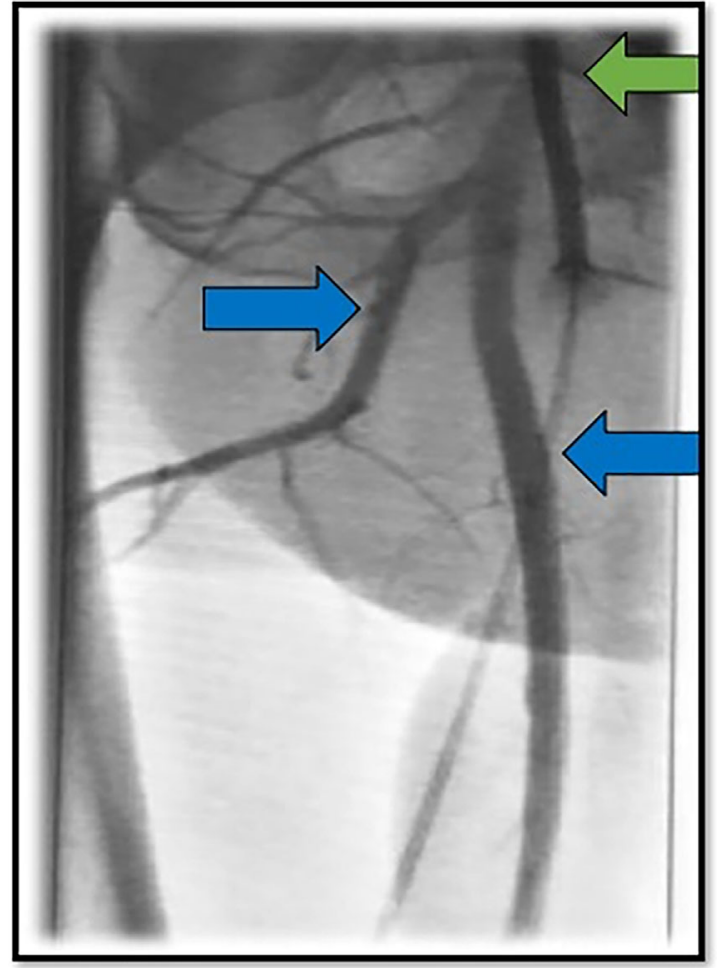

FIGURE 2 Right ilio-femoral angiogram with Impella $14 \mathrm{Fr}$ sheath (green arrow) showing adequate flow into the superficial femoral artery and deep femoral arteries (orange arrows) around the largebore sheath

catheter engagement into the ipsilateral iliac artery from an additional arterial access. The extremities are able to tolerate low perfusion state for short periods of time (less than $30 \mathrm{~min}$ ). ${ }^{32}$ However, when limb perfusion is compromised it may be necessary to establish flow distally by ex-vivo ipsilateral bypass using antegrade access technique.

For patients presented to the cath lab with cardiogenic shock or elective high-risk coronary intervention, Impella 2.5 or CP have been the device of choice in the US. Impella 2.5 and CP provide direct cardiac pressure and volume unloading of the left ventricle and antegrade flow in the thoracic aorta of up to 2.5 and $4.0 \mathrm{~L} / \mathrm{min}$, respectively. The catheter-based device is typically inserted through a peripheral access using a single arterial access of $13 \mathrm{Fr}$ and $14 \mathrm{Fr}$, respectively. Impella has a specially designed two-step peel away sheath with a tapered shaft (14French base to 9-French tip) that allows for adequate blood flow even in smaller caliber ilio-femoral arterial vessels. Once Impella device is positioned in place, the peel-away sheath then removed and repositioning sheath is advanced to the access site.

\section{6 | Reperfusion techniques}

PVD and CAD have similar risk factors, and thus, it is common to encounter the challenge of treating structural or complex coronary patients with significant concomitant PAD. ${ }^{14,15,33}$ Small caliber common femoral and iliac arteries with severe PAD significantly increase the risk for vascular complications. That risk increases greatly as the larger bore sheaths are used., ${ }^{6,16-18}$ 
Insertion of large bore sheath may result in complete vessel occlusion and acute limb ischemia that may threaten limb viability. It is therefore important to ensure adequate perfusion to the limb by careful assessment of the iliofemoral artery prior to insertion of large bore sheath. A challenging clinical scenario often encountered in practice is a patient who is hemodynamically dependent on a shortterm mechanical support device and whose large bore sheath is found to be occlusive. Several interventions can be performed in the setting of an occlusive sheath to restore perfusion to the distal lower extremity in such patients.

\subsection{1 | Peel away sheath}

The Impella mechanical support device comes with a specially designed 2-step peel away sheath with a tapered shaft (14 Fr base to $9 \mathrm{Fr}$ tip) that allows for adequate blood flow even in smaller caliber ilio-femoral arterial vessels. In case of an occlusive sheath compromising limb perfusion, peeling away the $14 \mathrm{Fr}$ introducer sheath leaves the Impella with the smaller $9 \mathrm{Fr}$ repositioning sheath which may be sufficient to restore limb perfusion. One potential complication of this technique is catheter migration. To minimize this complication, two people should carry out this intervention under direct fluoroscopic guidance. Specifically, one operator stabilizes the Impella to avoid any pullback while the other operator peels away the external sheath. The repositioning sheath can then be re-advanced through the arteriotomy site. This maneuver, however, may lead to increased bleeding as the sheath size is tapered down.

\subsection{2 | External contralateral bypass circuit}

If there is occlusion of the external iliac artery and CFA by the large bore sheath, the ipsilateral superficial femoral artery is accessed via a 4 or $5 \mathrm{Fr}$ short sheath with a micro puncture kit in antegrade fashion. A contralateral CFA access is then obtained with a $6 \mathrm{Fr}$ sheath. Then the side arm of the contralateral sheath is connected to the side arm of the ipsilateral antegrade sheath using a male-to-male connector. The result is an external femoral-femoral bypass whereby blood flows from the contralateral $6 \mathrm{Fr}$ sheath through the side arm into the side arm of the ipsilateral $5 \mathrm{Fr}$ antegrade sheath down the ischemic limb providing adequate perfusion (Figures $3 \mathrm{~A}$ and $3 \mathrm{~B}$ ). In situation when occlusion of flow is anticipated (ie, ECMO sheath), antegrade access with 5 or $6 \mathrm{Fr}$ sheath can be placed preemptively prior to the large bore sheath insertion. The target activated clotting time (ACT) should be higher than standard to maintain the flow (range 200-220 s). Also, hourly serial Doppler ultrasound assessment of the lower extremity pulsations is recommended

\subsection{3 | External ipsilateral bypass circuit}

An alternative strategy utilizes the sidearm of the large bore occlusive sheath to create an ipsilateral bypass circuit. A 4 to $5 \mathrm{Fr}$ short sheath is inserted in the ipsilateral superficial femoral artery with a micro puncture kit in antegrade fashion. The side arm of the ipsilateral
(A)



(B)

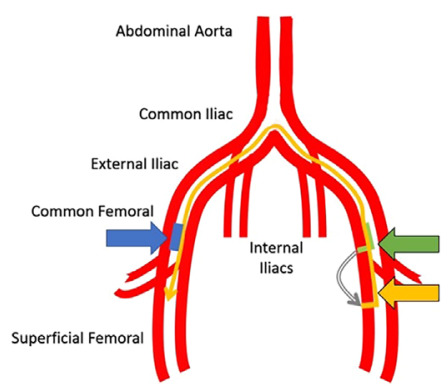

FIGURE 3 A, Contralateral bypass circuit showing the side arm of the contralateral common femoral artery sheath connected to the side arm of the ipsilateral antegrade sheath via long extension tubing and a male-to-male connector providing perfusion to the superficial femoral artery. B, Figure depicting the contralateral bypass circuit. Large occlusive sheath in the right common femoral artery. Blood flows through the left $7 \mathrm{Fr}$ sheath (green) to the $4 \mathrm{Fr}$ daughter sheath (yellow) and through the $45 \mathrm{~cm}$ catheter to the contralateral side. The catheter then passes along the $14 \mathrm{Fr}$ sheath (blue) and to the right SFA

antegrade sheath can then be connected via a male-to-male connector to the side arm of the large bore occlusive sheath constituting an ipsilateral bypass circuit that provides adequate perfusion to the lower limb. When using this strategy with an Impella device, the repositioning sheath must not be advanced into the Impella 14-Fr sheath to avoid occlusion of the side arm that is now providing perfusion (Figures $4 \mathrm{~A}$ and $4 \mathrm{~B}$ ). The management of this conduit follows the same protocol as that outlined above for the contralateral bypass technique. The advantage of this strategy is that it does not require a contralateral arterial access.

\subsection{4 | Internal contralateral bypass circuit}

The superficial femoral artery may be diseased and totally occluded, not an uncommon finding in patients with CAD, precluding antegrade superficial femoral artery access. In such cases, internal contralateral femoral to profunda bypass might be an alternative option to maintain perfusion to the ischemic limb. This is achieved by first inserting a $7 \mathrm{Fr}$ sheath in the contralateral CFA. Through this sheath, a $5 \mathrm{Fr}$ catheter (IMA, OMNI flush) is advanced and selectively engaged into the ipsilateral common iliac artery. A 0.035 hydrophilic wire 
(A)



(B)



FIGURE 4 A, Ipsilateral bypass circuit showing the side arm of the large bore sheath connected to the side arm of the antegrade sheath via a male-to-male connector providing distal perfusion. B, Figure depicting another technique for ipsilateral bypass circuit. Distal to the Impella sheath a $5 \mathrm{Fr}$ sheath was used to access the ipsilateral common femoral artery. The side arm of the Impella and $5 \mathrm{Fr}$ sheath are connected using male-to-male connector creating a continuous flow from Impella sheath (blue) to the side arm of the $5 \mathrm{Fr}$ sheath located in the SFA (green)

(Terumo Glidewire ${ }^{\circledR}$ guidewire) is then advanced through this system across the aorto-iliac bifurcation, past the occlusive large bore sheath and beyond the arteriotomy site into the ipsilateral profunda femoris artery in case of SFA occlusion. The $5 \mathrm{Fr}$ catheter is then exchanged for a $4 \mathrm{Fr}, 45-55 \mathrm{~cm}$ long sheath that is advanced over the wire into the ipsilateral profunda femoris. By connecting the side arm of the $7 \mathrm{Fr}$ contralateral sheath and that of the $4 \mathrm{Fr}, 45-55 \mathrm{~cm}$ sheath using a male-to-male connector, a bypass circuit is created whereby blood flows from the contralateral femoral artery via the connected side arms, through the cross-over $4 \mathrm{Fr}$ sheath into the ipsilateral profunda femoris artery distal to the occlusive sheath providing sufficient perfusion to maintain limb viability (Figures 5 and 6). The management of this conduit follows the same protocol as that outlined above for the contralateral and the ipsilateral bypass techniques with anticoagulation for a target ACT range of 200-220 s.

\section{7 | Sheath removal and hemostasis}

Hemostasis after removal of small arterial sheaths is often achieved with manual compression, or with arterial closure devices that are readily available. Larger sheaths have been associated with worse outcome due to bleeding and vascular complications. ${ }^{6}$ Removal of

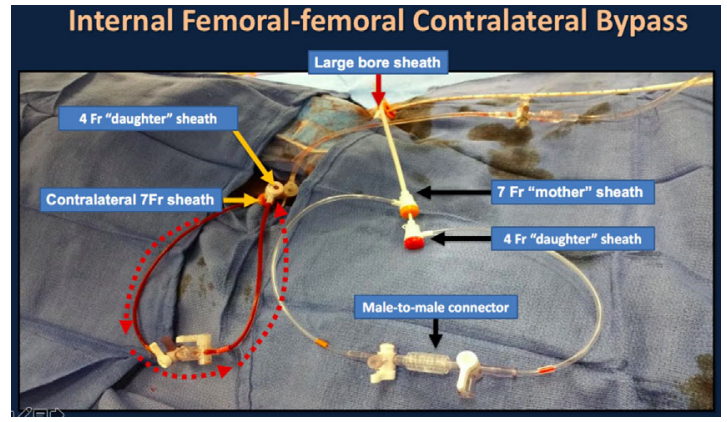

FIGURE 5 Internal contralateral femoral to ipsilateral profunda femoris bypass circuit showing the side arm of the contralateral (Right) $7 \mathrm{Fr}$ femoral sheath connected to the side arm of the $4 \mathrm{Fr}$ 45-55 cm cross-over sheath via a male-to-male connector providing perfusion to the ipsilateral (Left) profunda femoris artery distal to the occlusive (Left) large sheath

large-bore sheaths requires meticulous technique to prevent major or even life-threatening bleeding complications and to assure vessel patency. Manual compression of large arteriotomy is not the ideal technique due to lack of direct visualization and poor control of the puncture site. To date, there are no FDA-approved closure devices designed for large size arterial punctures. Perclose ProGlide system (Abbott Vascular Devices, CA) is a suture-mediated closure device designed to close 5-8 French access sites. ${ }^{30,31}$ It is possible to use more than one suture and to pre-deploy the suture before upsizing to a large-bore sheath as described above. Complete deployment of the Perclose sutures is performed when the large-bore sheath is removed. It is recommended to perform large-bore sheath removal in the cardiac catheterization laboratory which provides best controlled environment. While in the cath lab, one operator can apply manual pressure above the access point and pull the large sheath, while the second operator deploying the closure device. In certain cases when the risk of bleeding is high (anticoagulation, calcified vessel), we recommend dry closure technique. Dry closure is achieved by advancing $6-9 \times 40 \mathrm{~mm}$ peripheral balloon proximal to the access site. Once the balloon is inflated the sheath then removed and PerClose device is deployed fully and sutures snitched to the arteriotomy site..$^{30,31}$

\subsection{1 | Vascular closure device}

Increased use of large-bore sheaths for structural heart procedures and advanced MCS devices introduced the practice of preclosure with Perclose VCD. Preclosure is done with partial deployment of suturemediated VCD after the artery is accessed with a small caliber sheath and before insertion of the large-bore arterial sheath. Once the procedure is done, preclosure will facilitate hemostasis once the largebore sheath is removed. Observational data demonstrated that percutaneous approach to TAVR using VCD with preclosure is associated with similar vascular outcomes to surgical cut-down for arterial access, but with shorter length of stay. ${ }^{34-36}$ Routine use of VCD for large bore access might reduce bleeding and vascular complications, and facilitate patient ambulation and decrease hospital 




FIGURE 6 Hemodynamic tracing of external iliac artery via Impella $14 \mathrm{Fr}$ sheath side arm during endovascular temporary $40 \times 9 \mathrm{~mm}$ balloon inflation. Tracing illustrates blunting of pulse pressure (blue arrow) that confirm adequate temporary internal occlusion of the common femoral artery

length of stay. Despite increasing experience with VCD, complications of VCD include infection, embolization, and device failure leading to ischemia or access-site bleeding. VCD-related complications increase with lack of proficiency with the selected device. ${ }^{37}$ Several new large caliber VCD are in development for structural heart interventions, and are undergoing clinical investigation. ${ }^{38}$

For patients treated with Impella and require delayed hemostasis, the closure system needs to be sterilized, and secured especially if MCS is indicated for several days. Our practice is to sterilize the Perclose sutures, snare knot pusher, and suture trimmer with chlorhexidine gluconate (ChloraPrep ${ }^{\circledR}$ ) and use Tegaderm to keep them separate and away from the access site. We then re-sterilize the site with ChloraPrep and wrap all Perclose parts with sterile towels. ${ }^{39}$ We keep the Perclose sutures under the Tegaderm for 2-3 days on average. The duration depends on severity of current shock and time to recovery.

\subsection{2 | Temporary endovascular balloon tamponade}

The temporary endovascular balloon tamponade or "dry field closure technique," is a useful technique that prevents extensive bleeding at the time of large-bore access closure. It allows the operator to have control of the access site while deploying VCD especially in patients who are at increased risk for bleeding (ie, on anticoagulation, access site calcification, vascular tortuosity). From a 7-French sheath in the contralateral CFA, a 5Fr catheter (IMA, OMNI flush) is advanced and selectively engaged into the ipsilateral common iliac artery. Then, a 0.035 hydrophilic stiff wire (Terumo Glide Advantage wire) is advanced into the ipsilateral CFA, where the large-bore sheath is inserted. The wire is further advanced around the large sheath and distal to the arteriotomy site into the superficial femoral artery. Afterwards, the sheath and the catheter are exchanged into $45-55 \mathrm{~cm}$ long sheath. The long sheath is then advanced to the level of the ipsilateral external iliac artery. The large-bore sheath is withdrawn to the level of the distal segment of the external iliac artery. Subsequently, an $8-9 \mathrm{~mm}$ diameter $\times 20-40 \mathrm{~mm}$ long balloon (size of the balloon is determined by femoral angiogram) is inflated to low pressure (2-4 ATM) to temporarily occlude flow in the external iliac artery proximal to the large-bore sheath. To monitor effective occlusion, a pressure transducer is connected to the large-bore sheath side branch. With effective balloon inflation, the pressure wave form flattens, and the pulse pressure diminishes (Figure 7). The large-bore sheath is then removed from the body and the partially deployed Perclose ProGlide sutures seal the puncture site. Once this step is done, the occlusive balloon tamponade slowly deflated to restore blood flow to the access leg. If residual bleeding is present after Perclose ProGlide sutures deployment, then same balloon is advanced at the arteriotomy and inflated to provide endovascular low-pressure tamponade until hemostasis is achieved. Protamine Sulfate can be used to reverse anticoagulation when hemostasis is not achieved.

Dry field technique can be used in emergency situations when the large-bore sheath is inserted quickly without Perclose ProGlide sutures to expedite MCS support. When it is appropriate to remove the largebore sheath, prolonged endovascular balloon tamponade (30-60 min) may be sufficient to achieve hemostasis. Rarely, the arteriotomy site may continue to bleed after this intervention. In that case, a covered stent can be deployed at the arteriotomy site to achieve hemostasis.

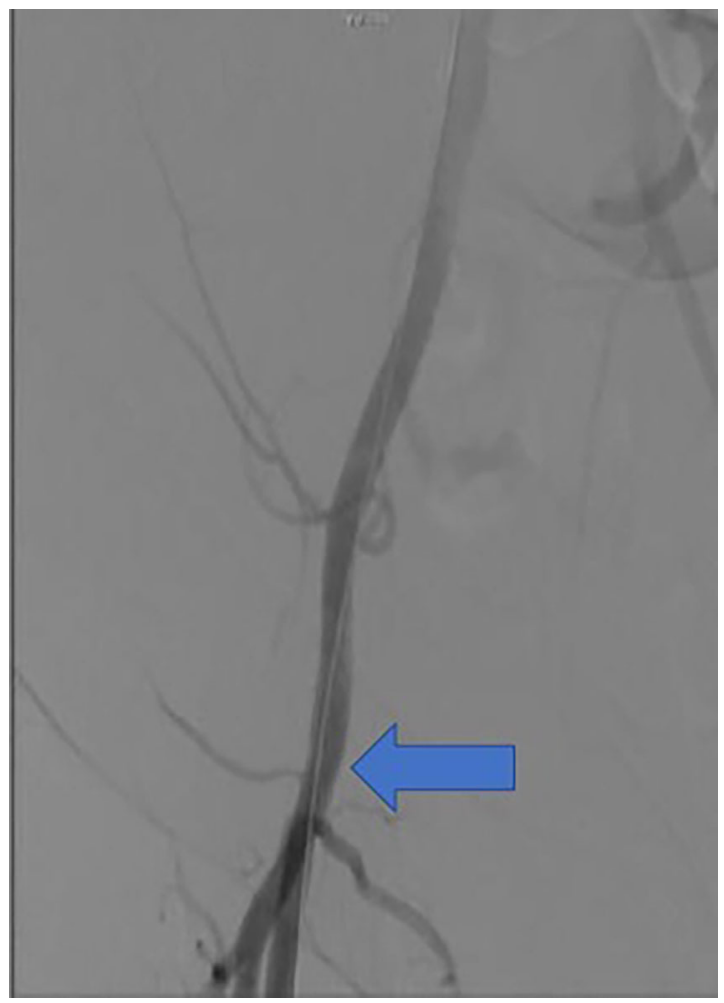

FIGURE 7 Right iliac, right common femoral artery, and bifurcation showing post procedure intact vessels with complete hemostasis. The arrow indicates the access site 
Once hemostasis is achieved, the recommendation is to confirm vessel patency and rule out sheath-related complications such as vessel dissection, occlusion, thrombosis by performing a final angiogram using a pigtail in the descending aorta or contralateral femoral catheter (Figure 8). VIABAHN® (GORE $®)$ is self-expandable covered stents are indicated for iliac and superficial femoral artery complications. Six millimeter diameter stents can be delivered via 6-French arterial sheath while 7 and $8 \mathrm{~mm}$ stents are requires 7-French arterial sheath. iCast on the otherhand is a balloon-expandable stent that is available in diameters of $5-12 \mathrm{~mm}$ and delivered via 6 or 7-French introducer sheath.

\section{8 | Complication management}

\subsection{1 | Acute limb ischemia}

Acute limb ischemia in the setting of large-bore access can be due to complete vessel occlusion (when vessel diameter is $<5.0 \mathrm{~mm}$ ), arterial dissection or thromboembolism. Utilization of a femoral-femoral bypass circuit, as described above, may prevent these complications. Close monitoring to ensure adequate limb perfusion with hourly clinical and Doppler assessment of distal extremity pulses is indicated when the sheath is in place. Serial biomarker evaluation (plasma lactate) is recommended as a surrogate for inadequate limb perfusion and tissue necrosis. Thromboembolism or plaque embolization are not uncommon complications. ${ }^{6}$ Thrombus or debris may flow downstream causing
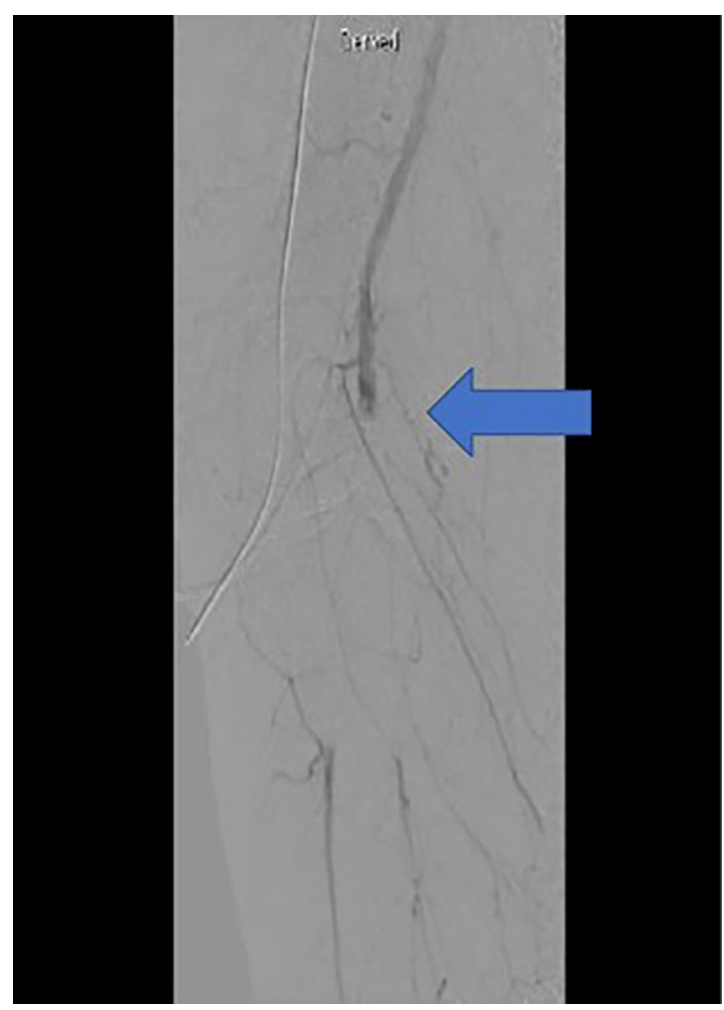

FIGURE 8 Digital subtraction angiography of the right SFApopliteal and trifurcation shows acute arterial thrombosis occluding the right popliteal artery (arrow) occlusion of the tibio-peroneal trifurcation causing acute limb ischemia. After sheath removal, it is recommended to perform complete angiography of the arteriotomy site and runoff to the tibio-peroneal trifurcation at the end of procedure to assure distal vessels patency.

\subsection{2 | Vascular pseudoaneurysm}

In a patient with a recent history of femoral artery puncture the presence of pain, swelling, and a palpable mass in the groin should prompt further investigation. The mass may be pulsatile with a thrill or bruit. Complications can arise due to mass effect. Arteriotomy below the CFA bifurcation is associated with an increased risk of local hematoma and pseudoaneurysm formation owing to the lack of the posterior anchoring of manual compression provided by the head of the femur. Other risk factors for pseudoaneurysm include: multiple arterial punctures, and large-bore sheath usage. ${ }^{40-42}$ Meticulous access technique as presented should preclude from multiple arterial punctures and suboptimal access location. Nevertheless, even with the best technique pseudoaneurysms may still occur. Pseudoaneurysms are commonly diagnosed clinically and confirmed with ultrasound imaging. The pseudoaneurysm can be treated in multiple ways. To illustrate, the majority of small pseudoaneurysms (less than $2-3 \mathrm{~cm}$ ) arising from arterial puncture are known to thrombose spontaneously within 4 weeks and thus conservative management is appropriate. A second option of treatment is percutaneous ultrasoundguided thrombin injection into the pseudoaneurysm. Thrombin converts fibrinogen into fibrin. Fibrin is then cross-linked by factor XIlla in the presence of calcium leading to the formation of thrombus. The final option is surgical repair. Rapid expansion, rupture, infection, and mass effect resulting in distal or cutaneous ischaemia or peripheral neuropathy are indications for surgery. ${ }^{43-45}$

\subsection{3 | Bleeding}

The femoral artery is a large-caliber vessel with diameter ranging from 8 to $9 \mathrm{~mm}$ in normal adults which accommodate large sheaths. ${ }^{6,46}$ The femoral artery enters the retroperitoneal space before joining the iliac artery. If the arteriotomy site is too high, this may lead to a retroperitoneal hematoma. This is a rare complication of femoral artery access, but is associated with high mortality. ${ }^{47}$ It is imperative to have high level of suspicion for these complications and develop a protocol for prompt identification and management. The first step is to prevent ongoing extravasation. This is achieved by inflating a balloon at low pressure to cover the site of perforation. While bleeding is controlled, a covered stent is rapidly deployed. Simultaneously, a vascular surgeon should be notified and made ready for possible emergent transfer of the patient for emergency surgery in the event percutaneous management of this complication fails.

\section{2 | CONCLUSION}

Arterial access and hemostasis are fundamental aspects of cardiac catheterization and intervention. In recent years, large-bore sheaths 
for structural heart procedures and advanced MCS devices have been used more frequently. Emerging approaches to arteriotomy, and newgeneration VCD have a role in reducing vascular complications and bleeding associated with large-bore access procedure and accelerates the time to hemostasis and ambulation.

\section{CLINICAL PERSPECTIVES}

Despite the increasing use large-bore femoral accesses, there are no unified protocols to guide the large-bore femoral technique. This article provides strategies for placement, removal, and complication management to serve as a guide for interventional cardiologists who use large-bore femoral arterial access and includes several techniques that were developed over the last decade at our center with the experience of thousands of large-bore femoral arterial procedures.

\section{AUTHORS CONTRIBUTIONS}

Each one of the authors listed contributed to multiple components of the paper.

\section{CONFLICTS OF INTEREST}

None.

\section{ORCID}

Nimrod Blank iD http://orcid.org/0000-0003-1993-4244

M. Chadi Alraies iD http://orcid.org/0000-0002-7874-4566

\section{REFERENCES}

1. Radner S. An attempt at the roentgenologic visualization of coronary blood vessels in man. Acta Radiologica. 1945;26:497-502.

2. Radner S. Thoracal aortography by catheterization from the radlal artery. Acta Radiologica. 1948;29:178-180.

3. Brodén B, Jönsson G, Karnell J. Thoracic aortography: observations on technical problems connected with the method and various risks involved in its use. Acta Radiologica. 1949:498-508.

4. Webb JG, Chandavimol M, Thompson CR, et al. Percutaneous aortic valve implantation retrograde from the femoral artery. Circulation. 2006;113:842-850

5. Howell M, Villareal R, Krajcer Z. Percutaneous access and closure of femoral artery access sites associated with endoluminal repair of abdominal aortic aneurysms. J Endovasc Ther. 2001;8:68-74.

6. Redfors B, Watson BM, McAndrew T, et al. Mortality, length of stay, and cost implications of procedural bleeding after percutaneous interventions using large-bore catheters. JAMA Cardiol. 2017;2: 798-802.

7. Harold JG, Bass TA, Bashore TM, et al. ACCF/AHA/SCAI 2013 update of the clinical competence statement on coronary artery interventional procedures. Catheter Cardiovasc Interv. 2013;82:2.

8. Levine GN, Bates ER, Blankenship JC, et al. ACC/AHA/SCAl focused update on primary percutaneous coronary intervention for patients with ST-elevation myocardial infarction: an update of the 2011 ACCF/
AHA/SCAI guideline for percutaneous coronary intervention and the 2013 ACCF/AHA guideline for the management of ST-elevation myocardial infarction. Circulation. 2015;CIR.0000000000000336.

9. Archbold RA, Robinson NM, Schilling RJ. Radial artery access for coronary angiography and percutaneous coronary intervention. BMJ. 2004;329:443.

10. O'neill WW, Kleiman NS, Moses J, et al. A prospective randomized clinical trial of hemodynamic support with impella $2.5 \mathrm{TM}$ versus intraaortic balloon pump in patients undergoing high-risk percutaneous coronary intervention: the PROTECT II study. Circulation. 2012; CIRCULATIONAHA. 112.098194

11. Werdan K, Gielen S, Ebelt H, Hochman JS. Mechanical circulatory support in cardiogenic shock. Eur Heart J. 2013;35:156-167.

12. Pedersen OM, Aslaksen A, Vik-Mo H. Ultrasound measurement of the luminal diameter of the abdominal aorta and iliac arteries in patients without vascular disease. J Vasc Surg. 1993;17:596-601.

13. Pearce WH, Slaughter MS, LeMaire $S$, et al. Aortic diameter as a function of age, gender, and body surface area. Surgery. 1993;114: 691-697.

14. Gerhard-Herman MD, Gornik HL, Barrett C, et al. 2016 AHA/ACC guideline on the management of patients with lower extremity peripheral artery disease: executive summary: a report of the American College of Cardiology/American Heart Association Task Force on Clinical Practice Guidelines. Circulation. 2017;135: e686-e725.

15. Montalescot G, Sechtem U, Achenbach S, et al. 2013 ESC guidelines on the management of stable coronary artery disease: the task force on the management of stable coronary artery disease of the European Society of Cardiology. Eur Heart J. 2013;34:2949-3003.

16. Judkins MP, Gander MP. Prevention of complications of coronary arteriography. Circulation. 1974;49:599-602.

17. Samal AK, White CJ. Percutaneous management of access site complications. Catheter Cardiovasc Interv. 2002;57:12-23.

18. Sanghvi K, Kurian D, Coppola J. Transradial intervention of iliac and superficial femoral artery disease is feasible. J Interv Cardiol. 2008; 21:385-387.

19. Kiemeneij F, Laarman GJ, Odekerken D, Slagboom T, van der Wieken R. A randomized comparison of percutaneous transluminal coronary angioplasty by the radial, brachial and femoral approaches: the access study. J Am Coll Cardiol. 1997;29:1269-1275.

20. Walther T, Simon P, Dewey $T$, et al. Transapical minimally invasive aortic valve implantation. Circulation. 2007;116:I-240-I-245.

21. Coburn MA. A Perspective on Sheath Selection and Access Sites for Coronary Angiography. In. Cath Lab Digest. Vol 18: HMP Cardiovascular; Sep. 2010

22. Htyte N, White CJ. Vascular access for percutaneous interventions and angiography. In: Mukherjee D, Bates ER, Roffi M, Moliterno DJ, eds. Cardiovascular Catheterization and Intervention: A Textbook of Coronary, Peripheral, and Structural Heart Disease. 1st ed. London: Informa Healthcare; 2010.

23. Bauernschmitt R, Schreiber C, Bleiziffer S, et al. Transcatheter aortic valve implantation through the ascending aorta: an alternative option for no-access patients. Paper presented at: The heart surgery forum, 2009.

24. Greenbaum AB, O'Neill WW, Paone G, et al. Caval-aortic access to allow transcatheter aortic valve replacement in otherwise ineligible patients: initial human experience. J Am College Cardiol. 2014;63:2795-2804.

25. Abu-Fadel MS, Sparling JM, Zacharias SJ, et al. Fluoroscopy vs traditional guided femoral arterial access and the use of closure devices: a randomized controlled trial. Catheter Cardiovasc Interv. 2009;74:533-539.

26. Fitts J, Ver Lee P, Hofmaster P, Malenka D. Fluoroscopy-guided femoral artery puncture reduces the risk of $\mathrm{PCl}$-related vascular complications. J Interv Cardiol. 2008;21:273-278.

27. Seto AH, Abu-Fadel MS, Sparling JM, et al. Real-time ultrasound guidance facilitates femoral arterial access and reduces vascular 
complications: fAUST (Femoral Arterial Access With Ultrasound Trial). JACC. 2010;3:751-758.

28. Hind D, Calvert N, McWilliams R, et al. Ultrasonic locating devices for central venous cannulation: meta-analysis. BMJ. 2003;327:361.

29. Ramlawi B, Anaya-Ayala JE, Reardon MJ. Transcatheter aortic valve replacement (TAVR): access planning and strategies. Methodist DeBakey Cardiovasc J. 2012;8:22-25.

30. Martin JL, Pratsos A, Magargee E, et al. A randomized trial comparing compression, perclose proglide ${ }^{\mathrm{TM}}$ and Angio-Seal VIP ${ }^{\mathrm{TM}}$ for arterial closure following percutaneous coronary intervention: the cap trial. Catheter Cardiovasc Interv. 2008;71:1-5.

31. Dosluoglu HH, Cherr GS, Harris LM, Dryjski ML. Total percutaneous endovascular repair of abdominal aortic aneurysms using Perclose ProGlide closure devices. J Endovasc Ther. 2007;14:184-188.

32. Lukasiewicz A. Treatment of acute lower limb ischaemia. Vasa. 2016;45:213-221.

33. Kurra V, Schoenhagen P, Roselli EE, et al. Prevalence of significant peripheral artery disease in patients evaluated for percutaneous aortic valve insertion: preprocedural assessment with multidetector computed tomography. J Thorac Cardiovasc Surg. 2009;137:1258-1264.

34. Toggweiler S. Percutaneous aortic valve replacement: vascular outcomes with a fully percutaneous procedure.

35. Durand E, Borz B, Godin M, et al. Transfemoral aortic valve replacement with the Edwards SAPIEN and Edwards SAPIEN XT prosthesis using exclusively local anesthesia and fluoroscopic guidance: feasibility and 30-day outcomes. JACC. 2012;5:461-467.

36. Abdul-Jawad Altisent O, Durand E, Muñoz-García AJ, et al. Warfarin and antiplatelet therapy versus warfarin alone for treating patients with atrial fibrillation undergoing transcatheter aortic valve replacement. JACC Cardiovasc Interv. 2016;9:1706-1717.

37. Resnic FS, Wang TY, Arora N, et al. Quantifying the learning curve in the use of a novel vascular closure device: an analysis of the NCDR (National Cardiovascular Data Registry) CathPCI registry. JACC. 2012;5:82-89.

38. Van Mieghem NM, Latib A, van der Heyden J, et al. Percutaneous plugbased arteriotomy closure device for large-bore access: a multicenter prospective study. JACC. 2017;10:613-619.
39. Lata K, Htun WW, Grines C, Kaki A, Schreiber T. Pre-close technique of percutaneous closure for delayed hemostasis of large-bore femoral sheaths. Catheter Cardiovasc Interv. 2016;87:S165.

40. Sherev DA, Shaw RE, Brent BN. Angiographic predictors of femoral access site complications: implication for planned percutaneous coronary intervention.

41. Hamraoui K, Ernst SM, van Dessel PF, et al. Efficacy and safety of percutaneous treatment of iatrogenic femoral artery pseudoaneurysm by biodegradable collagen injection. J Am College Cardiol. 2002;39: 1297-1304.

42. Hessel SJ, Adams DF, Abrams HL. Complications of angiography. Radiology. 1981;138:273-281.

43. Altin R, Flicker $\mathrm{S}$, Naidech $\mathrm{H}$. Pseudoaneurysm and arteriovenous fistula after femoral artery catheterization: association with low femoral punctures. Am J Roentgenol. 1989;152:629-631.

44. Ahmad F, Turner S, Torrie P, Gibson M. latrogenic femoral artery pseudoaneurysms-a review of current methods of diagnosis and treatment. Clin Radiol. 2008;63:1310-1316.

45. Morgan R, Belli A-M. Current treatment methods for postcatheterization pseudoaneurysms. J Vasc Interv Radiol. 2003;14:697-710.

46. Lee MS, Applegate B, Rao SV, Kirtane AJ, Seto A, Stone GW. Minimizing femoral artery access complications during percutaneous coronary intervention: a comprehensive review. Catheter Cardiovasc Interv. 2014;84:62-69.

47. Pitta SR, Prasad A, Kumar G, Lennon R, Rihal CS, Holmes DR. Location of femoral artery access and correlation with vascular complications. Catheter Cardiovasc Interv. 2011;78:294-299.

How to cite this article: Kaki A, Blank N, Alraies MC, et al. Access and closure management of large bore femoral arterial access. J Interv Cardiol. 2018;31:969-977. https://doi.org/10.1111/joic.12571 